\title{
Literature reviews are not all the same
}

\author{
Amanda C de C Williams ${ }^{\mathrm{i}}$
}

Dear Editor,

I was very interested in the discussion in the Editorial of the first 2017 issue of Torture Journal which referred to two similar literature reviews with opposite conclusions (Weiss et al., 2016; Patel, Williams, \& Kellezi, 2016; Patel, Kellezi, \& Williams, 2014) and would like to clarify and elaborate some of the differences, which I think are of relevance to the conclusions.

There are two approaches to review. Narrative review selects according to explicit or implicit criteria from studies found by systematic or idiosyncratic search, then proceeds by taking the results of the included studies at face value. This holds even when the included trials are underpowered or uninterpretable, or their results are impossible to differentiate from effects that are not specific to the therapeutic method (anything from the passage of time to interest and concern from the research team). Narrative review authors look for simple majorities across their trial set to report on outcome, disregarding the strong bias in publication towards studies that show positive effects rather than no effects of therapy, the suppression of negative studies where authors or funders had an interest in propagating the (usually drug) treatment, and the tendency of underpowered trials to

${ }^{i} \mathrm{PhD}, \mathrm{CPsychol}$; Reader in Clinical Health Psychology, University College London; Research Consultant, International Centre for Health \& Human Rights; and, Consultant Clinical Psychologist, University College London NHS Foundation Trust show treatment benefit. Narrative reviews frequently disagree, even when their constituent studies overlap very substantially, and because this led to long delays in instituting effective, even life-saving, treatments in medicine, systematic review was developed to provide a cumulative summary of all studies that met reasonable criteria for scientific rigour, and to use a shared and accountable method that enabled widespread sharing of data, updating, and further research within review topics (4).

Systematic review and meta-analysis selects by explicit criteria from systematic search, usually only RCTs which it combines in meta-analysis for maximum power, and quantifying the size of effects of treatment, the confidence we can have in those effects, and the likelihood that the findings could be easily overturned by, for instance, discovering unpublished negative trials. The evident advantage over narrative reviews, and the consensus on conclusions that followed the use of transparent and accountable methods, led to the establishment of the worldwide Cochrane Collaboration. Further, among systematic reviews and meta-analyses, Cochrane reviews are the most reliable and least subject to error (Chalmers I, Altman DG, 1995).

Weiss et al. (2016) used systematic search and explicit criteria, which considerably strengthens their narrative review, but did not restrict their review to RCTs which, whatever their limitations, at least provide interpretable results by having a comparable untreated or differently treated group. Such a 
comparison group is very important, since nonspecific or placebo effects are a helpful component of any treatment or apparent treatment. Patients who feel that someone is listening to them, asking sensible questions, discussing possible treatments and outcomes, and even expending resources on their account, may well become more hopeful, less anxious, and evaluate their symptoms and problems differently. These effects can be seen as an asset rather than a nuisance, and a reminder to us to do all we can to strengthen those processes of listening, empathy, engagement, and shared agenda with the patient. But because at least some of those processes happen in control arms of trials, it does seem important to identify whether technique-based treatment offers more, which is why it is so hard to interpret uncontrolled studies. This is not to suggest that randomised group studies are the only way forward: we underuse single case studies where people are their own control.

So while the Weiss et al. (2016) review provides very valuable data on types of treatment, on targets of treatment, and on evaluation methods in a wide range of included studies, it is not equipped to provide an overall estimate of effectiveness of those treatments, unlike the Cochrane review (2014) and our summary of it for Torture Journal (2016). Thus the apparent contradiction is easily resolved by taking from each review what it provides using appropriate methodology.

\section{References}

Chalmers I, Altman DG (eds). Systematic reviews. London, BMJ Publishing Group, 1995

Page MJ, Shamseer L, Altman DG, Tetzlaff J, Sampson M, Tricco AC, Catalá-López F, Li L, Reid EK, Sarkis-Onofre R, Moher D. Epidemiology and reporting characteristics of systematic reviews of biomedical research: a crosssectional study. PLoS Med 13(5): e1002028. doi:10.1371/journal.pmed.1002028.
Patel N, Williams ACdeC, Kellezi B. Reviewing outcomes of psychological interventions with torture survivors : conceptual, methodological and ethical issues. Torture J 2016;26(1):2-16.

Patel N, Kellezi B, Williams ACDC. Psychological, social and welfare interventions for psychological health and well-being of torture survivors. Cochrane Database Syst Rev 2014, Issue 11. Art. No.: CD009317. DOI: 10.1002/14651858. CD009317.pub2.

Weiss WM, Ugueto AM, Mahmooth Z, Murray LK, Hall BJ, Nadison M, et al. Mental health interventions and priorities for research for adult survivors of torture and systematic violence: a review of the literature. Torture 2016;26(1):17-45. 